Pacific Journal of Mathematics

CHARACTERIZATIONS OF ARBOROIDS AND DENDRITIC
SPACES THOMAS BOURQUE MUENZENBERGER,
RAYMOND EARL SMITHSON AND L. E. WARD 


\title{
CHARACTERIZATIONS OF ARBOROIDS AND DENDRITIC SPACES
}

\author{
T. B. Muenzenberger, R. E. Smithson, \\ AND L. E. WARD, JR.
}

In this paper several characterizations of arboroids, arcwise connected dendritic spaces, and a related, wider class of spaces which we call weakly nested are obtained. For example, it is shown that an arcwise connected Hausdorff space is dendritic if and only if it is uniquely arcwise connected and each connected subspace is arcwise connected. These characterizations give considerable insight into the internal structure of such spaces. Also a number of characterizations of topological intervals and trees are given, and an interesting embedding theorem for weakly nested spaces is proved.

1. Introduction. We begin by recalling a few definitions. A continuum is a compact, connected Hausdroff space. A space $X$ is orderable if it admits a total order $\leqq$ such that all sets of the form $\{x \in X: x<a\}$ and $\{x \in X: a<x\}$ generate the topology, where $a \in X$. It is well-known that an orderable space is completely normal and Hausdorff [1]. An arc is a nondegenerate orderable continuum, or, what is the same thing, a continuum with exactly two noncut points, which are called the endpoints of the arc. This definition is a departure from and generalization of the classical usage in which an arc is separable. A space is arcwise connected if each two distinct points are the endpoints of some arc contained in the space, and it is uniquely arcwise connected if that arc is unique. A connected space is hereditarily unicoherent if the intersection of any pair of its closed connected subsets is connected.

A connected space is said to be dendritic if each pair of distinct points can be separated by some third point. Note that a dendritic space is Hausdorff [2]. A tree is a compact dendritic space, and hence a metrizable tree is a dendrite.

Knaster has coined the term dendroid to mean a metrizable continuum which is hereditarily unicoherent and arcwise connected [3]. Following [14] we use the term arboroid for the Hausdorff analog of a dendroid. That is, an arboroid is a continuum which is hereditarily unicoherent and arcwise connected. Although many properties of dendroids carry over directly to arboroids, it is a frequently noted phenomenon that nonmetrizable continua may exhibit pathological qualities not found in the metrizable case. For example, even with the hypothesis of local connectivity, a continuum may fail 
to be arcwise connected and may fail to be the continuous image of an arc [5]. The study of dendritic spaces, arboroids, and similar objects is complicated in much the same fashion.

2. Characterizations of arboroids. An arcwise connected Hausdorff space is nested if the union of each nest of arcs is contained in an arc, or, equivalently, the closure of the union of each nest of arcs is an arc. A simple closed curve is the union of two arcs having only their endpoints in common, or, what is the same thing, a nondegenerate continuum in which each doubleton subset is a separator.

THEOREM 1. A space is an arboroid if and only if it is a uniquely arcwise connected continuum in which each subcontinuum is arcwise connected.

Proof. Suppose first that $X$ is an arboroid. If $X$ is not uniquely arcwise connected, then, by the argument used to prove Lemma 2.2.14 in [7], $X$ contains a simple closed curve, contradicting the hereditary unicoherence of $X$. To see that subcontinua are arcwise connected, let $x$ and $y$ be distinct elements of a subcontinuum $Y$. If $A$ is the unique arc in $X$ with endpoints $x$ and $y$, then $A \cap Y$ is a connected subset of $A$ containing $x$ and $y$, and so $A=A \cap Y \subseteq Y$.

Conversely, suppose that $X$ is a uniquely arcwise connected continuum in which each subcontinuum is arcwise connected. Any two subcontinua are arcwise connected, and thus their intersection is also arcwise connected since $X$ is uniquely arcwise connected.

THEOREM 2. A space is an arboroid if and only if it is a nested continuum in which each subcontinuum is arcwise connected.

Proof. It is known that any arboroid is nested [9] and that any nested space is uniquely arcwise connected [7].

Similar characterizations of dendroids were obtained earlier in [4].

3. Characterizations of arcwise connected dendritic spaces. A topological interval is a nondegenerate connected orderable space. Certainly an arc is just a compact topological interval, and, conversely, the addition, if necessary, of least and greatest elements to a topological interval leads to a compactification which is an arc [6]. The latter fact will follow from subsequent results in the present paper. The topological intervals can also be characterized as being the nondegenerate connected subspaces of arcs. An arcwise connected 
Hausdorff space is weakly nested if the closure of the union of each nest of ares is a topological interval. Clearly any nested space is weakly nested, and, conversely, a compact weakly nested space is nested. The following lemma may be found in [1] and [2].

LEMMA 1. If $H$ is a connected subspace of a connected space $X$, then the following hold.

(a) If $X-H=A \cup B$ where $A$ and $B$ are separated sets, then $A \cup H$ is connected.

(b) If $C$ is a component of $X-H$, then $X-C$ is connected.

TheOREM 3. A space is arcwise connected and dendritic if and only if it is a uniquely arcwise connected Hausdorff space in which each connected subspace is arcwise connected.

Proof. Suppose first that $X$ is arcwise connected and dendritic. Then each connected subspace of $X$ is arcwise connected by Proposition 6.1 in [15], and $X$ is uniquely arcwise connected by Proposition 2.2 and Lemma 17.1 in [15] or by the proof of Theorem 14 in [15].

Conversely suppose that $X$ is a uniquely arcwise connected Hausdorff space in which each connected subspace is arcwise connected. To show that $X$ is dendritic, let $x$ and $y$ be distinct points in $X$ and let $[x, y]$ denote the unique arc with endpoints $x$ and $y$. Let $z \in[x, y]-\{x, y\}$. Then $X-\{z\}$ is disconnected since it does not contain $[x, y]$. Therefore $X-\{z\}=A \cup B$ where $A$ and $B$ are nonempty separated sets. If $x \in A$ and $y \in B$ or vice versa, then $z$ separates $x$ and $y$. So we may assume that $x, y \in A$. Let $C_{x}$ be the component of $A$ containing $x$. Then $y \in A-C_{x}$ since $[x, y]$ is not contained in $C_{x}$. Suppose now that $C_{x} \cap \overline{A-C}_{x} \neq \varnothing$, and select $x_{0} \in C_{x} \cap \overline{A-C_{x}}$. Now $A \cup\{z\}$ is connected by Lemma 1 (a), and so $A \cup\{z\}-C_{x}$ is connected by Lemma $1(\mathrm{~b})$. Hence $\left(A-C_{x}\right) \cup\left\{x_{0}, z\right\}$ is connected and therefore arcwise connected. Therefore $\left[x_{0}, z\right] \subseteq$ $\left(A-C_{x}\right) \cup\left\{x_{0}, z\right\}$. However $\left[x_{0}, z\right] \subseteq A \cup\{z\}$ since the latter set is arcwise connected. So $\left[x_{0}, z\right]-\{z\} \cong A$, whence $\left[x_{0}, z\right]-\{z\} \cong C_{x}$, a contradiction. It therefore follows that $C_{x} \cap \overline{A-C_{x}}=\varnothing$, and $X-$ $\{z\}=C_{x} \cup\left[\left(A-C_{x}\right) \cup B\right]$ is a separation of $x$ and $y$ by $z$. Hence $X$ is dendritic.

At this point it is helpful to introduce the order structure available in a uniquely arcwise connected Hausdorff space. A family $\mathscr{C}$ of subsets of a set $X$ is said to provide $X$ with the chain structure of a mod provided the following five conditions are met.

C1. For all $x, y \in X$ there exists an element of $\mathscr{C}$ containing $x$ and $y$. 
C2. If $\varnothing \neq \mathscr{C}_{0} \subseteq \mathscr{C}$ and if $\bigcap \mathscr{C}_{0} \neq \varnothing$, then $\bigcap \mathscr{C}_{0} \in \mathscr{C}$. Define the chain with endpoints $x$ and $y$ by the formula $C[x, y]=$ $\bigcap\{C \in \mathscr{C}: x, y \in C\}$, and let $C(x, y)=C[x, y]-\{x, y\}$ and $C[x, y)=$ $C[x, y]-\{y\}$. A set $B \subseteq X$ is chainable if $x, y \in B$ implies $C[x, y] \subseteq B$.

C3. For all $C \in \mathscr{C}$ there exists a unique pair $x, y \in X$ such that $C=C[x, y]$.

C4. The union of two intersecting chains is chainable.

C5. If $x, y \in X$ and $x \neq y$, then $C(x, y) \neq \varnothing$.

If $\mathscr{C}$ provides $X$ with the chain structure of a mod and $e \in X$, then the chain order $\leqq$ with basepoint $e$ is defined by the rule: $x \leqq y$ if and only if $x \in C[e, y]$.

A subset $\leqq$ of $X \times X$ is said to provide $X$ with the order structure of a mod provided the following five conditions are met.

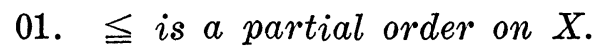

02. There is a least element $e$ in $X$.

03. If $x \in X$, then $L(x)=\{y \in X: y \leqq x\}$ is totally ordered.

04. Every nonempty subset of $X$ which is bounded above (below) has a supremum (an infimum).

05 . $\leqq i s$ order dense.

If $\leqq$ provides $X$ with the order structure of a mod, then the chain $[x, y]$ with endpoints $x$ and $y$ can be defined as follows where $M(x)=\{y \in X: x \leqq y\}$ and $x \wedge y=\inf \{x, y\}$.

$$
[x, y]= \begin{cases}L(y) \cap M(x) & \text { if } x \leqq y . \\ {[x \wedge y, x] \cup[x \wedge y, y]} & \text { if } x \text { and } y \text { are not comparable } .\end{cases}
$$

Suppose now that $\mathscr{C}$ provides $X$ with the chain structure of a mod and that $\leqq$ is the chain order with basepoint $e$ defined by means of the chains in $\mathscr{C}$. Then $\leqq$ provides $X$ with the order structure of a mod, and the chains defined in terms of $\leqq$ satisfy

$$
[x, y]=C[x, y] \text { for all } x, y \in X .
$$

All of this was proved in [8].

Conversely suppose that $\leqq$ provides $X$ with the order structure of a mod and that the chains $[x, y]$ are defined in terms of $\leqq$. Then $\mathscr{C}=\{[x, y]: x, y \in X\}$ provides $X$ with the chain structure of a mod [8], and the chains defined in terms of $\mathscr{C}$ satisfy

$$
C[x, y]=[x, y] \text { for all } x, y \in X \text {. }
$$

We say that a set $X$ admits the structure of a mod if there is a family $\mathscr{C}$ which provides $X$ with the chain structure of a mod, or, equivalently, there is a partial order $\leqq$ which provides $X$ with the order structure of a mod. More simply we say that $(X, \mathscr{C})$ or $(X, \leqq)$ is a mod. The main point here is that once $X$ admits the structure 
of a mod in either form, the chains and the chain order are completely determined.

BUtTERFly Lemma. Suppose that $(X, \leqq)$ is a mod containing $p, q, x$, and $y$ where $x$ and $y$ are not comparable. If $x \leqq p$ and $y \leqq q$, then $x \wedge y=p \wedge q$.

Proof. Since $x \wedge y$ is a lower bound for $p$ and $q$, it follows that $x \wedge y \leqq p \wedge q$. For the reverse inequality, observe that $x, p \wedge q \leqq p$, and hence $x$ and $p \wedge q$ are comparable. If $x \leqq p \wedge q$, then $x, y \leqq q$ which implies that $x$ and $y$ are comparable, a contradiction. So $p \wedge q \leqq x$ and similarly $p \wedge q \leqq y$, whence $p \wedge q \leqq x \wedge y$.

Let $X$ be a uniquely arcwise connected Hausdorff space. For each pair of distinct points $x$ and $y$ in $X$, let $A[x, y]$ denote the unique arc in $X$ with endpoints $x$ and $y$. Put $A[x, x]=\{x\}$ for $x \in X$.

Lemma 2. A uniquely arcwise connected Hausdorff space admits the structure of a mod.

Proof. We show that $\mathscr{C}=\{A[x, y]: x, y \in X\}$ provides $X$ with the chain structure of a mod. $\mathrm{C} 1$ is clear since $X$ is arcwise connected. $\mathrm{C} 2$ follows since $X$ is uniquely arcwise connected, since arcs are arcwise connected, and since any subcontinuum of an arc is an arc or a point [7]. Observe that for $x, y \in X, A[x, y]=C[x, y]=$ $\bigcap\{C \in \mathscr{C}: x, y \in C\}$, for if $x, y \in A[x, y] \in \mathscr{C}$, then $C[x, y] \subseteq A[x, y]$. On the other hand, $x, y \in C \in \mathscr{C}$ implies $A[x, y] \subseteq C$ since $C$ is arcwise connected. Next note that $B \cong X$ is chainable if and only if $B$ is arcwise connected. C3 follows since any arc has exactly two noncutpoints. C4 follows since the union of two intersecting arcs is arcwise connected, and C5 follows since any arc is nondegenerate. Thus $(X, \mathscr{C})$ is a mod.

Now let $e \in X$ and let $\leqq$ be the chain order with basepoint $e$; that is, $x \leqq y$ if and only if $x \in A[e, y]$. By our earlier remarks $\leqq$ provides $X$ with the order structure of a mod, and the chains defined in terms of $\leqq$ satisfy the following equality.

$$
A[x, y]=\left\{\begin{aligned}
L(y) \cap M(x) & \text { if } x \leqq y \\
A[x \wedge y, x] \cup & A[x \wedge y, y] \\
& \text { if } x \text { and } y \text { are not comparable } .
\end{aligned}\right.
$$

Alternate proofs of the sufficiency of the conditions in Theorems 1 and 3 can be constructed by verifying that the chain order satisfies the conditions listed in Theorem 1 in [13] and Theorem 11 in [15]. The chain order can also be used to prove the following theorem in 
which we have simplified the notation by writing $[x, y]$ for $A[x, y]$.

THEOREM 4. A space is arcwise connected and dendritic if and only if it is a weakly nested space in which each connected subspace is arcwise connected.

Proof. Suppose first that $X$ is a weakly nested space in which each connected subspace is arcwise connected. Then $X$ is uniquely arcwise connected. For otherwise $X$ would contain a simple closed curve $S$, and one could construct a nest of arcs in $S$, the closure of the union of which would be all of $S$ (see the proof of Lemma 2.2.14 in [7]), contradicting the hypothesis that $X$ is weakly nested. Thus $X$ is uniquely arcwise connected, and Theorem 3 applies.

Suppose conversely that $X$ is arcwise connected and dendritic. By Theorem 3, $X$ is a uniquely arcwise connected Hausdorff space in which each connected subspace is arcwise connected. To show that $X$ is weakly nested, we let $\mathscr{N}$ be a nest of arcs in $X$ and use the mod structure developed above. Suppose that $[x, y] \in \mathscr{N}$ where $x$ and $y$ are not comparable and that $[p, q]$ is any arc in $\mathscr{N}$. If $[x, y] \subseteq[p, q]$, then equality (1) above implies that $p$ and $q$ are not comparable and that, without loss of generality, $x \leqq p$ and $y \leqq q$. The Butterfly Lemma then implies that $x \wedge y=p \wedge q$. On the other hand, if $[p, q] \subseteq[x, y]$, then $x \wedge y \leqq p \wedge q$ by similar reasoning. By defining

$$
a=\inf \{p \wedge q:[p, q] \in \mathscr{N}\},
$$

we may assume without loss of generality that $\mathscr{N}=\left\{\left[a, q_{\lambda}\right]: \lambda \in \Lambda\right\}$ where $a \leqq q_{\lambda}$ for each $\lambda \in \Lambda$. Now $M(x)$ is closed for each $x \in X$ by Theorem 4.8 in [10] and Theorem 3 in [15], but this is easy enough to prove directly here. Suppose that $p \in \overline{M(x)}-M(x)$. Observe that $M(x)=\bigcup\{[x, y]: y \geqq x\}$ is connected, and so $M(x) \cup\{p\}$ is connected and therefore arcwise connected. Thus $\varnothing \neq(x \wedge p, x) \subseteq[x, p] \subseteq$ $M(x) \cup\{p\}$, a contradiction. It follows that $M(x)$ is closed for each $x \in X$. Next observe that $\cup \mathscr{N}$ is a topological interval. For $\cup \mathscr{N}$ is obviously connected, and if $x \in \mathrm{U} \mathscr{N}$, then

$$
\begin{aligned}
& {[a, x)=(X-M(x)) \cap(\cup \mathscr{N}) \text { and }} \\
& (M(x)-\{x\}) \cap(\mathbf{U} \mathscr{N})=\cup \mathscr{N}-[a, x]
\end{aligned}
$$

are open in $U \mathscr{N}$. On the other hand, each open set in $U \mathscr{N}$ contains an open interval about each of its points since each arc in $\mathscr{N}$ has that property. Thus $U \mathscr{N}$ is orderable via the chain order, and so $U \mathscr{N}$ is a topological interval. We may assume that $\overline{\mathrm{U} \mathscr{N}} \neq \mathrm{U} \mathscr{N}$ and select $q \in \overline{\mathrm{U} \mathscr{N}}-\mathrm{U} \mathscr{N}$. We claim that $x \in \mathbf{U} \mathscr{N}$ implies $q \in M(x)$. For 
$q \in\left(\bigcup\left\{\left[a, q_{\lambda}\right]: \lambda \in \Lambda\right\}\right)^{-}=[a, x] \cup\left(\bigcup\left\{\left[x, q_{\lambda}\right]: x \leqq q_{\lambda}, \lambda \in \Lambda\right\}\right)^{-}$

implies $q \in\left(\bigcup\left\{\left[x, q_{2}\right]: x \leqq q_{\lambda}, \lambda \in \Lambda\right\}\right)^{-} \leqq M(x)$. Therefore $\mathbf{U} \mathscr{N} \subseteq$ $[a, q] \subseteq \widehat{\bigcup} \mathscr{N}$, and so $\overline{\mathrm{U} \mathscr{N}}=[a, q]$ is a topological interval.

The next result first appeared in [11] and follows from the proof of Theorem 4.

THEOREM 5. If $X$ is a uniquely arcwise connected Hausdorff continuum, if $\leqq$ is a chain order, and if $M(x)$ is closed for each $x \in X$, then $X$ is a nested continuum.

4. Characterizations of trees, arcs, and topological intervals. The next result follows from Theorems 3 and 4 .

THEOREM 6. The following are equivalent for a space $X$.

(a) The space $X$ is a tree.

(b) The space $X$ is a uniquely arcwise connected continuum in which each connected subspace is arcwise connected.

(c) The space $X$ is a nested continuum in which each connected subspace is arcwise connected.

A space $X$ is atriodic if and only if $X$ does not contain three arcs each having a point $a$ as a common endpoint and not intersecting otherwise. It was proved in [9] that a space $X$ is an arc if and only if $X$ is atriodic and nested.

LEMMA 3. A uniquely arcwise connected space $X$ is atriodic if and only if the complement of each point has at most two arc components.

Proof. If $\mathrm{U}_{n=1}^{3}\left[a, a_{n}\right]$ is a triod in $X$, then $a_{1}, a_{2}$, and $a_{3}$ lie in distinct arc components of $X-\{a\}$. Conversely, if $X-\{a\}$ has at least three arc components, then select $a_{1}, a_{2}$, and $a_{3}$ in different arc components of $X-\{a\}$ and observe that $\bigcup_{n=1}^{3}\left[a, a_{n}\right]$ is a triod in $X$.

THEOREM 7. The following are equivalent for a nondegenerate Hausdorff space $X$.

(a) The space $X$ is a topological interval.

(b) The space $X$ is atriodic, uniquely arcwise connected, and contains at most two noncutpoints.

(c) The space $X$ is atriodic, uniquely arcwise connected, and each connected subspace is arcwise connected.

(d) The space $X$ is atriodic, arcwise connected, and the intersection of each pair of connected subspaces is connected. 
(e) The space $X$ is atriodic, arcwise connected, and dendritic.

(f) The space $X$ is atriodic and weakly nested.

Proof. $\quad(a) \Rightarrow$ (b) A topological interval is clearly uniquely arcwise connected, and it is atriodic by Lemma 3 . The only possible noncutpoints are the greatest and least elements if they exist.

(b) $\Rightarrow$ (c) If $C$ is a connected subspace of $X$ and if $a_{1}, a_{2} \in C$ such that $\left[a_{1}, a_{2}\right] \nsubseteq C$, then any element of $\left(a_{1}, a_{2}\right)$ is a noncutpoint of $X$.

$(c) \Leftrightarrow(d)$ See the proof of Theorem 1 .

(c) $\Rightarrow$ (e) Theorem 3 .

(e) $\Rightarrow$ (f) Theorem 4 .

(f) $\Rightarrow$ (a) Suppose that $X$ is atriodic and weakly nested. Then $X$ is uniquely arcwise connected, and it is easy to see that $X$ cannot contain three noncomparable elements (with respect to the chain order). Thus $X$ can be written as the union of a nest of arcs, and $X$ is therefore a topological interval.

By specializing to continua, one obtains similar characterizations of ares.

\section{Examples.}

EXAMPLE 1. Let $T=\{(x, 0): 0<x \leqq 1\}, X_{0}=\{(0, y): 0 \leqq y \leqq 1\}$, $X_{n}=\{(1 / n, y): 0 \leqq y \leqq 1\}$ for $n \geqq 1$, and let $C=T \cup \bigcup\left\{X_{n}: n \geqq 0\right\}$. With the Euclidean subspace topology, the closed infinite comb $C$ is an arboroid which contains a connected subspace that is not arcwise connected, and so $C$ is not dendritic.

EXAMPLE 2. Let $S=\{(x, y): 0<x \leqq 1, y=\sin (\pi / x)\} \cup\{(0, y)$ : $-2 \leqq y \leqq 1\} \cup\{(x,-2): 0 \leqq x \leqq 1\} \cup\{(1, y):-2 \leqq y \leqq 0\}$. With the Euclidean subspace topology, the topologist's sine $1 / x$ circle $S$ is a uniquely arcwise connected, atriodic continuum which is neither dendritic, nor hereditarily unicoherent, nor nested. Every point is a noncutpoint, and $S$ contains a subcontinuum which is not arcwise connected.

ExAmple 3. Let $G=\{(x, y): 0<x \leqq 1, y=\sin (\pi / x)\} \cup\{(0,0)\}$. With the Euclidean subspace topology, the sine $1 / x$ graph $G$ is an atriodic dendritic space which is neither arcwise connected, nor weakly nested, nor a topological interval. The intersection of each pair of connected sets in $G$ is connected, and $G$ contains exactly two noncutpoints. Furthermore, the order topology induced by the cutpoint partial order is strictly contained in the Euclidean subspace topology. So $G$ is weakly orderable in the terminology of [2], but $G$ is not orderable (in our terminology). 
EXAMPLE 4. Let $M$ denote Mardešićs locally connected continuum which contains no proper locally connected subcontinuum [5]. The existence of this example depends on the Continuum Hypothesis. The cone over $M$, which we denote $C M$, is a locally connected nested continuum which is neither dendritic nor hereditarily unicoherent. Further, every point of $C M$ is a noncutpoint.

EXAMPLE 5. Example 12 in [15] is an arcwise connected dendritic space which is neither locally connected, nor locally compact, nor rim compact, nor semi-locally connected.

6. Characterizations of weakly nested spaces. The following order theoretic characterization of weakly nested spaces can be proven in a fashion similar to the proof of the characterization of nested spaces given in [12].

THEOREM 8. A Hausdorff space $X$ is weakly nested if and only if $X$ admits a partial order $\leqq$ which satisfies the following five conditions.

(a) There is a least element $e$ in $X$.

(b) The lower set $L(x)$ is compact and totally ordered for each $x \in X$.

(c) The partial order $\leqq$ is order dense.

(d) If $A$ is an arc in $X$, then any subset of $A$. which consists only of noncomparable elements can contain at most two points.

(e) Every maximal totally order subset of $M(\alpha)$ is closed for all $a \in X$.

We consider next the problem of embedding a weakly nested space in a nested space by adding, where necessary, the missing endpoints of maximal topological intervals. In general, this cannot be done in such a way that the containing space is Hausdorff.

Lemma 4. If $A$ is an arc in a space $X$ and $x \in \bar{A}-A$, then $A \cup\{x\}$ is not a topological interval.

Proof. If $A \cup\{x\}$ is orderable, then it is Hausdorff, and thus $A$ is closed in $A \cup\{x\}$ contrary to the assumption that $x \in \bar{A}-A$.

COROLlaRY. If $X$ is an arcwise connected space in which the closure of the union of each nest of arcs is a topological interval, then each arc in $X$ is closed in $X$, and so $X$ is uniquely arcwise connected.

Proof. If $A$ is an arc in $X$, then $\bar{A}$ is a topological interval 
by hypothesis, and hence $\bar{A}=A$ by Lemma 4 . If $A_{1}$ and $A_{2}$ are distinct arcs with the same endpoints, then $A_{1}-A_{2}$ is open in $A_{1}$, and we can construct a simple closed curve $S$ in $X$ as in [7].

EXAMPLE 6. Let $X_{1}=\{(x, 0): 0 \leqq x<1\}, X_{2}=\{(x, 0): 1<x \leqq 2\}$, $X_{3}=\{(1, y):-1 \leqq y \leqq 1\}$, and $X=X_{1} \cup X_{2} \cup X_{3}$. Neighborhoods of points $(x, 0)$ in $X_{1}$ or $X_{2}$ are the usual Euclidean neighborhoods in $X_{1}$ or $X_{2}$, respectively. Basic neighborhoods of points $\left(1, y_{0}\right)$ in $X_{3}$ are of the form

$$
\begin{aligned}
&\{(x, 0) \in X: 1-\varepsilon<x<1 \text { or } 1<x<1+\varepsilon\} \\
& \cup\left\{(1, y) \in X: y_{0}-\varepsilon<y<y_{0}+\varepsilon\right\}
\end{aligned}
$$

where $\varepsilon>0$. The space $X$ is arcwise connected, and the union of each nest of arcs in $X$ is contained in an arc. However, the closure of the union of a nest of arcs in $X$ need not be a topological interval. Further, $X$ is neither Hausdorff nor uniquely arcwise conncted.

We shall need the following lemma and construction in the proof of the next major result. In the material through Theorem 9, we assume that $X$ is a weakly nested space and that $\leqq$ is the chain order with basepoint $e$ defined in terms of the arcs in $X$. Then $(X, \leqq)$ is a mod by Lemma 2 , and, in fact, $\leqq$ satisfies the five conditions of Theorem 8. In particular, if $a \in X$ and if $T$ is a maximal totally ordered subset of $M(a)$, then $T$ is closed. Since $T$ is the union of a nest of arcs, it is a topological interval.

Lemma 5. If $A \subseteq X$ is a topological interval which is not totally ordered, then $\inf A \in A$.

Proof. That $\inf A$ exists in $X$ follows from the definition of a mod. Let $a_{1}, a_{2}$ be two points in $A$ which are not related and let $a_{0}=a_{1} \wedge a_{2}$. If $\inf A<a_{0}$, then there exists an $a \in A$ with $\inf A<a<a_{0}$. Since $A$ is a topological interval, $\left[a, a_{1}\right] \cup\left[a, a_{2}\right] \subseteq A$ but $\left[a, a_{1}\right] \cup\left[a, a_{2}\right]$ is a triod [9], contradicting Theorem 7. Thus $a_{0}=\inf A$. Finally, $a_{0} \in\left[a_{1}, a_{2}\right] \leqq A$, and so $\inf A \in A$.

A maximal totally ordered subset $T$ of $X$ is eventually in a subset $U$ of $X$ if there is $t_{0} \in T$ such that $\left\{t \in T: t_{0} \leqq t\right\} \subseteq U$. The set $T$ is frequently in $U$ if for each $t_{0} \in T$ it follows that $\left\{t \in T: t_{0}<t\right\} \cap$ $U \neq \varnothing$. Now let

$$
\begin{aligned}
\mathscr{C}= & \{T: T \text { is a maximal totally ordered subset of } X \text { containing } \\
& \text { no greatest element }\} .
\end{aligned}
$$

Then for each $T \in \mathscr{M}$ let $x_{T}$ be a distinct element not in $X$, and let $X^{*}=X \cup\left\{x_{T}: T \in \mathscr{C}\right\}$. Topologize $X^{*}$ by calling a subset $U$ 
open provided $U \cap X$ is open in $X$ and, for each $x_{T} \in U$, the interval $T$ is eventually in $U$. It is clear that $X$ is a dense open subset of $X^{*}$ and that $X^{*}$ is a $T_{1}$-space. Moreover, $X^{*}-X$ is a discrete subspace of $X^{*}$.

If $T \in \mathscr{N}$ and $a \in T$, let $\left[a, x_{T}\right)=\{x \in T: a \leqq x\}$. Then $\left[a, x_{T}\right)$ is a topological interval and $\overline{\left[a, x_{T}\right)}=\left[a, x_{T}\right) \cup\left\{x_{T}\right\}$. It is an easy exercise to verify that

$$
\left[a, x_{T}\right]=\left[a, x_{T}\right) \cup\left\{x_{T}\right\}
$$

is compact, connected, Hausdorff in its relative topology, and has exactly two noncutpoints; namely, $a$ and $x_{T}$. Thus $\left[a, x_{T}\right]$ is a closed arc, and hence $X^{*}$ is arcwise connected.

In the sequel, if $A$ is an arc in $X^{*}$ and $a, b \in A$, then $A[a, b]$ denotes the sub-arc of $A$ from $a$ to $b, A[a, b)=A[a, b]-\{b\}$, and $A(a, b)=A[a, b]-\{a, b\}$.

TheOREM 9. Any weakly nested space embeds as a dense open subspace of a uniquely arcwise connected $T_{1}$-space in which the closure of the union of each nest of arcs is an arc.

Proof. We have already shown that $X$ is a dense open subset of the space $X^{*}$ and that $X^{*}$ is arcwise connected. We shall show that $X^{*}$ satisfies the remaining conditions. Let $A$ be an arc in $X^{*}$. Since $X^{*}-X$ is discrete and closed, $A \cap\left(X^{*}-X\right)$ is compact and discrete-hence, finite. Now we need to show the following.

Claim 1. If $A$ is an arc in $X^{*}$ with endpoints $x_{T}$ and $a \in X$ and if $A\left[a, x_{T}\right) \subseteq X$, then $A\left[a, x_{T}\right)$ contains a least element in $X$.

If $A\left[a, x_{T}\right)$ is not totally ordered, then this claim follows from Lemma 5. On the other hand, if $A\left[a, x_{T}\right)$ is totally ordered, then $a$ is either the greatest element or the least element of $A\left[a, x_{T}\right)$. If $a$ is the greatest element of $A\left[a, x_{T}\right)$, then $L(a)$ is closed and contains $A\left[a, x_{T}\right)$. But this contradicts the fact that $x_{T} \in \overline{A\left[a, x_{T}\right)}$, and the claim is establised.

Now we prove another important fact about the $\operatorname{arcs}$ in $X^{*}$.

Claim 2. If $A$ is an arc in $X^{*}$ with endpoints $x_{T}$ and $a \in X$, if $A\left[a, x_{T}\right) \subseteq X$, and if $a_{0}$ is the least element of $A\left[a, x_{T}\right)$, then $A\left[a_{0}, x_{T}\right) \subseteq$ $T, T$ is eventually in $A\left[a_{0}, x_{T}\right)$, and $A\left[a_{0}, x_{T}\right)=\left[a_{0}, x_{T}\right)$.

Now $A\left[a_{0}, x_{T}\right) \subseteq M\left(a_{0}\right)$. Suppose there exist $b, c \in A\left[a_{0}, x_{T}\right)$ where $b$ and $c$ are not comparable. Then $A[b, c]=[b \wedge c, b] \cup[b \wedge c, c]$. If $a_{0}<b \wedge c$, then $A\left[a_{0}, x_{T}\right)$ contains the triod $\left[a_{0}, b \wedge c\right] \cup[b, c]$, which is impossible. So $a_{0}=b \wedge c$. But then $a_{0}$ is not an endpoint of $A\left[a_{0}, x_{T}\right)$, again a contradiction. Therefore $A\left[a_{0}, x_{T}\right)$ is contained in 
a maximal totally ordered subset $S$ of $X$. If $S \neq T$, then $(X-S) \cup$ $\left\{x_{T}\right\}$ is a neighborhood of $x_{T}$ and so $x_{T} \notin \overline{A\left[a_{0}, x_{T}\right)}$, a contradiction. So $A\left[a_{0}, x_{T}\right) \subseteq T$. To show that $T$ is eventually in $A\left[a_{0}, x_{T}\right)$, we show first that $T$ is frequently in $A\left[a_{0}, x_{T}\right)$. Suppose on the contrary that we can find a $t_{0} \in T$ such that $A\left[a_{0}, x_{T}\right) \cap\left\{t \in T: t_{0}<t\right\}=\varnothing$. Since $T$ is a topological interval, this implies that $A\left[a_{0}, x_{T}\right)$ is contained in a closed set disjoint from $\left\{t \in t: t_{0}<t\right\}$, and we again have a contradiction of the fact that $x_{T} \in \overline{A\left[a_{0}, x_{T}\right)}$. Thus $T$ is frequently in $A\left[a_{0}, x_{T}\right)$. Since $A\left[a_{0}, x_{T}\right)$ is a topological interval in $X, T$ is therefore eventually in $A\left[a_{0}, x_{T}\right)$ and, in fact, $A\left[a_{0}, x_{T}\right)=\left[a_{0}, x_{T}\right)$.

Claim 3. If $A$ is an arc in $X^{*}$ and $x_{T} \in A$, then $x_{T}$ is an endpoint of $A$.

Suppose that $x_{T}$ is not an endpoint of $A$. Since $A \cap\left(X^{*}-X\right)$ is finite, there exist $a, b \in A$ such that $x_{T} \in A(a, b)$ and $A[a, b]-\left\{x_{T}\right\} \subseteq$ $X$. Then $A[a, b]=A\left[a, x_{T}\right] \cup A\left[x_{T}, b\right]$ and $A\left[a, x_{T}\right) \cap A\left[b, x_{T}\right)=\varnothing$, whereas $T$ is eventually in both $A\left[a, x_{T}\right)$ and $A\left[b, x_{T}\right)$ by Claim 2. This is a contradiction.

We conclude that $A \cap\left(X^{*}-X\right)$ contains at most two points for any $\operatorname{arc} A$ in $X^{*}$ and that any such point is an endpoint of $A$. We now have a complete classification of the $\operatorname{arcs}$ in $X^{*}$. For if $A$ is an arc from $a$ to $b$, where $a, b \in X$, then $A \subseteq X$ by Claim 3, and hence $A$ is the unique arc in $X$ from $a$ to $b$. However, if $A$ is an arc from $a$ to $x_{T}$, where $a \in X$ and $x_{T} \in X^{*}-X$, then $A=$ $\left[a_{0}, a\right] \cup\left[a_{0}, x_{T}\right]$ by Claim 2 where $a_{0}$ is the least element of $A\left[a, x_{T}\right)$. Finally, let $A$ be an arc from $x_{S}$ to $x_{T}$. Then $A\left(x_{S}, x_{T}\right) \subseteq X$ by Claim 3. Observe that $A\left(x_{S}, x_{T}\right)$ is not totally ordered since $x_{S}, x_{T} \in \overline{A\left(x_{S}, x_{T}\right)}$. By Lemma 5 we may let $a_{0}=\inf A\left(x_{S}, x_{T}\right)$. Since $a_{0}$ is also the least element of both $A\left[a_{0}, x_{S}\right)$ and $A\left[a_{0}, x_{T}\right)$, we have $A=A\left[a_{0}, x_{S}\right] \cup$ $A\left[a_{0}, x_{T}\right]=\left[a_{0}, x_{S}\right] \cup\left[a_{0}, x_{T}\right]$ by Claim 2. It is easy to see that $a_{0}=$ sup $S \cap T$. It is now clear from this classification of ares that $X^{*}$ is uniquely arcwise connected and that each arc in $X^{*}$ is a closed set.

It remains to show that if $\mathscr{N}$ is a nest of arcs in $X^{*}$, then $\overline{\mathrm{U} \mathscr{N}}$ is an arc, and for this it is sufficient to show that $U \mathscr{N}$ is contained in an arc.

Since no arc in $X^{*}$ can contain more than two elements of $X^{*}-X$, the same is true of $\cup \mathscr{N}$. So if $x_{S}, x_{T} \in \mathbf{U} \mathscr{N}-X$, there is an arc $\left[x_{S}, x_{T}\right] \in \mathscr{N}$, and, by the above, $\left[x_{S}, x_{T}\right]$ is maximal in $\mathscr{N}$. Hence, $\cup \mathscr{N}=\left[x_{s}, x_{T}\right]$.

Now suppose that $U \mathscr{N}-X$ contains at most one element. We distinguish two cases.

Case 1. Suppose that $\cup \mathscr{N} \cap X$ is totally ordered. Then 
U $\mathscr{N} \cap X \subseteq T$, a maximal totally ordered set. Letting $a_{0}=$ inf $\bigcup \mathscr{N} \cap X$, it follows that $\cup \mathscr{N} \subseteq\left[a_{0}, t_{1}\right]$ or $U \mathscr{N} \subseteq\left[a_{0}, x_{T}\right]$, according as $T$ has or does not have a maximal element $t_{1}$.

Case 2. Suppose that $\cup \mathscr{N} \cap X$ is not totally ordered. Then there exists $A \in \mathscr{N}$ so that $A \cap X$ is not totally ordered. Then $A=A_{1} \cup A_{2}$ where $A_{1}$ and $A_{2}$ are arcs and $A_{1} \cap A_{2}=\left\{a_{0}\right\}$, where $a_{0}$ is the least element of $A \cap X$ (and hence the least element of $\cup \mathscr{N} \cap X)$. For each $B \in \mathscr{N}$ with $A \subseteq B$, there is a similar decomposition $B=B_{1} \cup B_{2}, B_{1} \cap B_{2}=\left\{a_{0}\right\}, A_{i} \subseteq B_{i}$, and we can apply the method of Case 1 to $\mathscr{N}_{1}=\left\{B_{1}: A \leqq B \in \mathscr{N}\right\}$ and $\mathscr{N}_{2}=\left\{B_{2}: A \leqq\right.$ $B \in \mathscr{N}\}$. Thus each of $U \mathscr{N}_{1}$ and $U \mathscr{N}_{2}$ is contained in an arc in $X^{*}$ where the containing arcs meet only in a common endpoint $a_{0}$.

Next we give an example to show $X^{*}$ need not be Hausdorff, and following that we give a condition which is sufficient to ensure that $X^{*}$ is Hausdorff.

EXAMPLE 7. Let $X=T \cup \cup\left\{X_{n}: n \geqq 1\right\}$ where $T$ and $X_{n}$ are as in Example 1. Let $x_{0}=(1,1)$. Basic neighborhoods of points $x \neq x_{0}$ in $X$ are the usual basic Euclidean neighborhoods in the plane. For $n \geqq 1$ let $X_{n}^{0}=\{(1 / n, y): 0<y<1\}$. Basic neighborhoods of $x_{0}$ are of the form

$$
\{(1, y): 1-\varepsilon<y \leqq 1\} \cup \bigcup\left\{X_{n}^{0}: n \geqq N\right\}
$$

where $0<\varepsilon \leqq 1$ and $N \geqq 1$. Now the space $X$ is weakly nested. However, if we give $X$ the chain order with basepoint $e=(1,0)$, then $T$ is the only maximal totally ordered subset of $X$ with no greatest element, and $x_{0}$ and $x_{T}$ cannot be separated by open sets in $X^{*}=X \cup\left\{x_{T}\right\}$.

Let $X$ be a weakly nested space and give $X$ the chain order with basepoint $e$. We say that $X$ is chain normal if the following two conditions are satisfied.

1. If $a \in X$ and $T$ is a maximal totally ordered subset of $X$ containing no greatest element, then there are disjoint open sets $U$ and $V$ in $X$ such that $a \in U$ and $T$ is eventually in $V$.

2. If $S$ and $T$ are distinct maximal totally ordered subsets of $X$ containing no greatest element, then there are disjoint open sets $U$ and $V$ in $X$ such that $S$ is eventually in $U$ and $T$ is eventually in $V$.

Since any maximal totally ordered subset of $X$ is closed, any normal weakly nested space is chain normal.

THEOREM 10. Any chain normal weakly nested space embeds as a dense open subspace of a nested space. 
A nested space containing a weakly nested space $X$ as a dense subspace is not in general unique even if $X$ is rim finite and dendritic.

EXAMPLE 8. Let $X$ be the set given in Example 7 together with the Euclidean subspace topology. Then $e(X)=X \cup\{(0,0)\}$ together with the Euclidean subspace topology is a nested space which contains $X$ as a dense subspace. Further, $X$ is rim finite and dendritic, and so $X$ admits a dendritic compactification $\delta(X)$ by Theorem 23 in [15]. The tree $\delta(X)$ is also a nested space which contains $X$ as a dense subspace. No two of the spaces $X^{*}, e(X)$, and $\delta(X)$ are homeomorphic.

Example 9. Let $M$ and $C M$ be as in Example 4. Consider $M$ to be the base of $C M$, select $x_{T} \in M$, and let $X=C M-\left\{x_{r}\right\}$. Observe that $X$ does not embed in $\delta(X)$ and that $X^{*}$ is not homeomorphic to $C M$.

Finally, we give a converse to the last theorem.

THEOREM 11. Any arcwise connected subspace of a nested space is weakly nested.

Proof. Let $Y$ be an arcwise connected subspace of a nested space $X$ and let $\mathscr{N}$ be a nest of ares in $Y$. Now $\bar{U} \mathscr{N}$ is an arc in $X$. So it suffices to prove that $C L_{Y}(\cup \mathscr{N})$ is connected. But this is evident since $C L_{Y}(\mathbf{U})=Y \cap \overline{\mathbf{U} \mathscr{N}}$ is the intersection of two arcwise connected sets.

\section{REFERENCES}

1. N. Bourbaki, General Topology, Addison-Wesley, Reading, 1966.

2. A. E. Brouwer, Treelike Spaces and Relxted Connected Topological Spaces, Mathematical Centre Tracts, 1977.

3. J. J. Charatonik, On ramification points in the classical sense, Fund. Math., 51 (1962), 229-252.

4. J. K. Harris, Order structures for certain acyclic topological spaces, Thesis, University of Oregon, 1962.

5. S. Mardešič, A locally connected cotinuum which contains no proper locally connected subcontinuum, Glasnik Mat. Ser. III, 2 (22) (1967), 167-168.

6. M. A. Maurice, Compact Ordered Spaces, Mathematical Centre Tracts, 1964.

7. L. Mohler, A fixed point theorem for continua which are hereditarily divisible by points, Fund. Math., 67 (1970), 345-358.

8. T. B. Muenzenberger and R. E. Smithson, Fixed point structures, Trans. Amer. Math. Soc., 184 (1973), 153-173.

9. —_ The structure of nested spaces, Trans. Amer. Math. Soc., 201 (1975), 57-87.

10. - Semilattice structures on dendritic spaces, Topology Proceedings, 2 (1977), 243-260.

11. R. E. Smithson, A note on acyclic continua, Colloq. Math., 19 (1968), 67-71. 
12. R. E. Smithson, An order theoretic characterization of nested spaces, to appear.

13. L. E. Ward, Jr., A fixed point theorem for multivalued functions, Pacific J. Math., 8 (1958), 921-927.

14. - Monotone surjections having more than one fixed point, Rocky Mtn. J. Math., 4 (1974), 95-106.

15. - Recent developments in dendritic spaces and related topics, Studies in Topology, 601-647, Academic Press, New York, 1975.

Received April 21, 1981.

KANSAS STATE UNIVERSITY

MANHATTAN, KS 66506

UNIVERSITY OF WYOMING

LARAMIE, WY 82071

AND

UNIVERSITY OF OREGON

EugENE, OR 97403 



\section{PACIFIC JOURNAL OF MATHEMATICS}

\section{EDITORS}

DONALD BABBITT (Managing Editor)

University of California

Los Angeles, California 90024

HUGO ROSSI

University of Utah

Salt Lake City, UT 84112

C. C. MOORE and ARTHur AGUS

University of California

Berkeley, CA 94720
J. DUGUNDJI

Department of Mathematics

University of Southern California

Los Angeles, California 90007

R. FINN and J. MILGRAM

Stanford University

Stanford, California 94305

\section{ASSOCIATE EDITORS}
R. Arnes
E. F. BECKENBACH
B. H. NeumanN
F. WOLF
K. YösHIDA

\section{SUPPORTING INSTITUTIONS}

UNIVERSITY OF ARIZONA

UNIVERSITY OF BRITISH COLUMBIA

CALIFORNIA INSTITUTE OF TECHNOLOGY

UNIVERSITY OF CALIFORNIA

MONTANA STATE UNIVERSITY

UNIVERSITY OF NEVADA, RENO

NEW MEXICO STATE UNIVERSITY

OREGON STATE UNIVERSITY
UNIVERSITY OF OREGON

UNIVERSITY OF SOUTHERN CALIFORNIA

STANFORD UNIVERSITY

UNIVERSITY OF HAWAII

UNIVERSITY OF TOKYO

UNIVERSITY OF UTAH

WASHINGTON STATE UNIVERSITY

UNIVERSITY OF WASHINGTON 


\section{Pacific Journal of Mathematics}

Vol. 102, No. $1 \quad$ January, 1982

S. Agou, Degré minimum des polynômes $f\left(\sum_{i=0}^{m} a_{i} X^{p^{r i}}\right)$ sur les corps finis

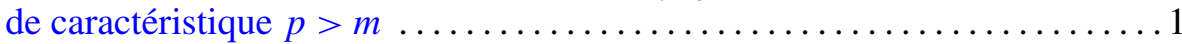

Chi Cheng Chen, On the image of the generalized Gauss map of a complete

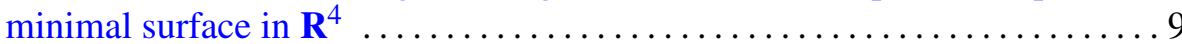

Thomas Curtis Craven and George Leslie Csordas, On the number of real

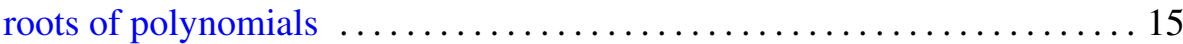

Allan L. Edelson and Kurt Kreith, Nonlinear relationships between oscillation and asymptotic behavior ....................... 29

B. Felzenszwalb and Antonio Giambruno, A commutativity theorem for

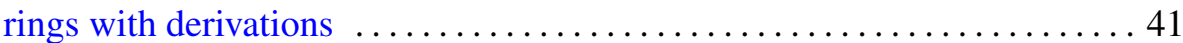

Richard Elam Heisey, Manifolds modelled on the direct limit of lines . . . . . 47

Steve J. Kaplan, Twisting to algebraically slice knots $\ldots \ldots \ldots \ldots \ldots \ldots 5$

Jeffrey C. Lagarias, Best simultaneous Diophantine approximations. II.

Behavior of consecutive best approximations $\ldots \ldots \ldots \ldots \ldots \ldots \ldots 61$

Masahiko Miyamoto, An affirmative answer to Glauberman's conjecture . . . 889

Thomas Bourque Muenzenberger, Raymond Earl Smithson and L. E.

Ward, Characterizations of arboroids and dendritic spaces ........... 107

William Leslie Pardon, The exact sequence of a localization for Witt

groups. II. Numerical invariants of odd-dimensional surgery

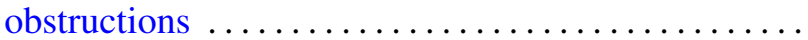

Bruce Eli Sagan, Bijective proofs of certain vector partition identities

Kichi-Suke Saito, Automorphisms and nonselfadjoint crossed products ...

John Joseph Sarraille, Module finiteness of low-dimensional PI rings ...

Gary Roy Spoar, Differentiable curves of cyclic order four . .

William Charles Waterhouse, Automorphisms of quotients of $\Pi \mathrm{GL}\left(n_{i}\right)$

Leslie Wilson, Mapgerms infinitely determined with respect to right-left equivalence

Rahman Mahmoud Younis, Interpolation in strongly logmodular

algebras 